\title{
Standard Triples of Structured Matrix
} Polynomials

Al-Ammari, Maha and Tisseur, Francoise

2011

MIMS EPrint: 2011.37

Manchester Institute for Mathematical Sciences

School of Mathematics

The University of Manchester

\footnotetext{
Reports available from: http://eprints.maths.manchester.ac.uk/

And by contacting: The MIMS Secretary

School of Mathematics

The University of Manchester

Manchester, M13 9PL, UK
} 


\title{
Standard Triples of Structured Matrix Polynomials
}

\author{
Maha Al-Ammari ${ }^{\mathrm{a}}$, Françoise Tisseur ${ }^{\mathrm{a}, 1, *}$ \\ ${ }^{a}$ School of Mathematics, The University of Manchester, Manchester, M13 9PL, UK
}

\begin{abstract}
The notion of standard triples plays a central role in the theory of matrix polynomials. We study such triples for matrix polynomials $P(\lambda)$ with structure $\mathcal{S}$, where $\mathcal{S}$ is the Hermitian, symmetric, $\star$-even, $\star$-odd, $\star$-palindromic or $\star$-antipalindromic structure (with $\star=*, T)$. We introduce the notion of $\mathcal{S}$-structured standard triple. With the exception of $T$-(anti)palindromic matrix polynomials of even degree with both -1 and 1 as eigenvalues, we show that $P(\lambda)$ has structure $\mathcal{S}$ if and only if $P(\lambda)$ admits an $\mathcal{S}$-structured standard triple, and moreover that every standard triple of a matrix polynomial with structure $\mathcal{S}$ is $\mathcal{S}$-structured. We investigate the important special case of $\mathcal{S}$-structured Jordan triples.
\end{abstract}

Keywords: standard triple, Jordan triple, structured matrix polynomial, Hermitian matrix polynomial, symmetric matrix polynomial, palindromic matrix polynomial, even matrix polynomial, odd matrix polynomial

\section{Introduction}

Standard and Jordan triples for matrix polynomials were introduced and developed by Gohberg, Lancaster and Rodman (see for example [4], [5], [6]). Jordan triples extend to matrix polynomials of degree $m$

$$
P(\lambda)=\sum_{j=0}^{m} \lambda^{j} A_{j}, \quad A_{j} \in \mathbb{F}^{n \times n}, \quad \operatorname{det}\left(A_{m}\right) \neq 0,
$$

the notion of Jordan pair $(X, J)$ for a single matrix $A \in \mathbb{C}^{n \times n}$, where $X \in \mathbb{C}^{n \times n}$ is nonsingular, $J$ is a Jordan canonical form for $A$, and $A=X J X^{-1}$. The matrix $X$ in a Jordan triple $(X, J, Y)$ for $P(\lambda)$ is $n \times m n$ and, as for the single matrix case, it contains the right eigenvectors and generalized eigenvectors of $P(\lambda)$. The matrix $J \in \mathbb{C}^{m n \times m n}$ is in Jordan canonical form, displaying the elementary divisors of $P(\lambda)$, and the matrix

\footnotetext{
ฟersion of May 10, 2011

* Corresponding author.

Email addresses: Maha.Al-Ammari@postgrad.manchester.ac.uk (Maha Al-Ammari), ftisseur@ma.man.ac.uk (Françoise Tisseur)

${ }^{1}$ The work of this author was supported by Engineering and Physical Sciences Research Council grant EP/I005293 and a Fellowship from the Leverhulme Trust.
} 
Table 1: Matrix polynomials $P(\lambda)=\sum_{j=0}^{m} \lambda^{j} A_{j}$ with structure $\mathcal{S} \in \mathbb{S}$.

\begin{tabular}{c|c|c} 
Structure $\mathcal{S}$ & Definition & Coefficients property \\
\hline Hermitian & $P(\lambda)=P^{*}(\lambda)$ & $A_{j}=A_{j}^{*}$ \\
symmetric & $P(\lambda)=P^{T}(\lambda)$ & $A_{j}=A_{j}^{T}$ \\
$\star$-even & $P(\lambda)=P^{\star}(-\lambda)$ & $A_{j}=(-1)^{j} A_{j}^{\star}$ \\
$\star$-odd & $P(\lambda)=-P^{\star}(-\lambda)$ & $A_{j}=(-1)^{j+1} A_{j}^{\star}$ \\
$\star$-palindromic & $P(\lambda)=\lambda^{m} P^{\star}\left(\frac{1}{\lambda}\right)$ & $A_{j}=A_{m-j}^{\star}$ \\
$\star$-antipalindromic & $P(\lambda)=-\lambda^{m} P^{\star}\left(\frac{1}{\lambda}\right)$ & $A_{j}=-A_{m-j}^{\star}$
\end{tabular}

$Y \in \mathbb{C}^{m n \times n}$ plays the role of $X^{-1}$ for a single matrix, i.e., the columns of $Y^{*}$ determine left eigenvectors and generalized eigenvectors of $P(\lambda)$. A Jordan triple is a particular standard triple $(U, T, V)$ in which the matrix $T$ is in canonical form. Standard and Jordan triples are defined precisely in section 2.2 .

Our objective is to study the standard and Jordan triples of structured matrix polynomials $P(\lambda)$ of the types listed in Table 1 , where we use $\star$ to denote the transpose $T$ for real matrices and either the transpose $T$ or the conjugate transpose $*$ for matrices with complex entries. The structure of standard and Jordan triples are well understood for Hermitian matrix polynomials [4], [5] and more recently real symmetric matrix polynomials [2], [12]. With no assumption on the sizes of the Jordan blocks, Gohberg, Lancaster and Rodman [4] show that if $(X, J, Y)$ is a Jordan triple for a Hermitian matrix polynomial then $Y=S X^{*}$ for some nonsingular $m n \times m n$ matrix $S$ such that $S=S^{*}$ and $J S=(J S)^{*}$. We show in section 3 that results of this type also hold for the structures in $\mathbb{S}$, where

$$
\begin{aligned}
\mathbb{S}=\{ & \text { Hermitian, symmetric, } * \text {-even, } * \text {-odd }, T \text {-even, } T \text {-odd }, \\
& \text { *-palindromic, } * \text {-antipalindromic, } T \text {-palindromic, } T \text {-antipalindromic }\} .
\end{aligned}
$$

For $\mathcal{S} \in \mathbb{S}$, we introduce the notion of $\mathcal{S}$-structured standard triples. With the exception of $T$-(anti)palindromic matrix polynomials of even degree with both -1 and 1 as eigenvalues, we show that $P(\lambda)$ has structure $\mathcal{S}$ if and only if $P(\lambda)$ admits an $\mathcal{S}$-structured standard triple, and that for any $P(\lambda)$ with structure $\mathcal{S}$, all standard triples for $P(\lambda)$ are $\mathcal{S}$-structured. Finally, we study in section 4 the special case of $\mathcal{S}$-structured Jordan triples.

Two important features of this work are (a) a distinction, when necessary, between triples and matrix polynomials defined over the complex $(\mathbb{C})$ or real $(\mathbb{R})$ fields, and (b) a unified presentation of the results, except in section 4 , where we provide explicit expressions for the $S$-matrix of $\mathcal{S}$-structured Jordan triples that are structure-dependent. 


\section{Preliminaries}

The set of all matrix polynomials with coefficient matrices in $\mathbb{F}^{n \times n}(\mathbb{F}=\mathbb{R}$ or $\mathbb{C})$ is denoted by $\mathcal{P}\left(\mathbb{F}^{n}\right)$. When the polynomials are structured with structure $\mathcal{S}$, the corresponding set is denoted by $\mathcal{P}_{\mathcal{S}}\left(\mathbb{F}^{n}\right)$ (see Table 1 ). Throughout this paper we assume that $P(\lambda)$ has a nonsingular leading coefficient matrix as in (1). Recall that $\lambda$ is an eigenvalue of $P(\lambda)$ with corresponding right eigenvector $x \neq 0$ and left eigenvector $y \neq 0$ if $P(\lambda) x=0$ and $y^{*} P(\lambda)=0$. We denote by $\Lambda(P)$ the set of eigenvalues of $P(\lambda)$.

\subsection{Structured linearizations}

Linearizations play a major role in the theory of matrix polynomials. They are $m n \times m n$ linear matrix polynomials $L(\lambda)=\lambda \mathcal{A}+\mathcal{B}$ related to $P(\lambda) \in \mathcal{P}\left(\mathbb{F}^{n}\right)$ of degree $m$ by

$$
E(\lambda) L(\lambda) F(\lambda)=\left[\begin{array}{cc}
P(\lambda) & 0 \\
0 & I_{(m-1) n}
\end{array}\right]
$$

for some matrix polynomials $E(\lambda)$ and $F(\lambda)$ with constant nonzero determinants. For example, the companion form

$$
\mathcal{C}=-\left[\begin{array}{cccc}
A_{m}^{-1} A_{m-1} & A_{m}^{-1} A_{m-2} & \ldots & A_{m}^{-1} A_{0} \\
-I_{n} & 0 & \ldots & 0 \\
& \ddots & \ddots & \vdots \\
0 & & -I_{n} & 0
\end{array}\right]
$$

of $P(\lambda)=\sum_{j=0}^{m} \lambda^{j} A_{j}$ defines a linearization $\lambda I-\mathcal{C}$ of $P(\lambda)$.

Some of the results in section 3 and all the results in section 4 rely on the construction of linearizations that preserve the structure of $P(\lambda) \in \mathcal{P}_{\mathcal{S}}\left(\mathbb{F}^{n}\right)$. The vector space of pencils

$$
\mathbb{L}_{1}(P)=\left\{L(\lambda): L(\lambda)\left(\Lambda \otimes I_{n}\right)=v \otimes P(\lambda), v \in \mathbb{F}^{m}\right\},
$$

introduced in [16], provides a rich source of such linearizations. Here $\Lambda=\left[\begin{array}{llll}\lambda^{m-1} & \ldots & \lambda & 1\end{array}\right]^{T}$. It is shown in [7], [13], [15] that for some $v \in \mathbb{F}^{m}$ satisfying the admissible constraint

(i) $v \in \mathbb{R}^{m}$ if $\mathcal{S}=$ Hermitian,

(ii) $v=\Sigma_{m} v$ if $\mathcal{S} \in\{T$-even, $T$-odd $\}$ or $v=\Sigma_{m} \bar{v}$ if $\mathcal{S} \in\{*$-even, $*$-odd $\}$,

(iii) $v=F_{m} v$ if $\mathcal{S} \in\{T$-palindromic, $T$-antipalindromic $\}$ or $v=F_{m} \bar{v}$ if $\mathcal{S} \in\{*$-palindromic, *-antipalindromic $\}$,

where

$$
\Sigma_{m}=\operatorname{diag}\left((-1)^{m-1}, \ldots,(-1)^{0}\right), \quad F_{m}=\left[\begin{array}{l}
\cdot^{\cdot} \\
1
\end{array}\right],
$$

there exists a unique pencil $\lambda \mathcal{A}_{\mathcal{S}}+\mathcal{B}_{\mathcal{S}} \in \mathbb{L}_{1}(P)$ with structure $\mathcal{S} \in \mathbb{S}$. This pencil is a linearization of $P(\lambda)$ if the roots of the v-polynomial

$$
\mathrm{p}(x ; v)=v_{1} x^{m-1}+v_{2} x^{m-2}+\cdots+v_{m-1} x+v_{m}
$$

are not eigenvalues of $P\left[16\right.$, Thm. 6.7]. The vector $v=e_{m}$, where $e_{m}$ is the $m$ th column of the $m \times m$ identity matrix, is an admissible vector for $\mathcal{S} \in$ \{Hermitian, 
symmetric, $\star$-even, $\star$-odd $\}$ since $e_{m} \in \mathbb{R}^{m}$ and $\Sigma_{m} e_{m}=e_{m}$. Also, the roots of $\mathrm{p}\left(x ; e_{m}\right)$ are all equal to $\infty$ and since $\operatorname{det}\left(A_{m}\right) \neq 0$ then $\infty \notin \Lambda(P)$. Hence the structured pencils $\lambda \mathcal{A}_{\mathcal{S}}+\mathcal{B}_{\mathcal{S}} \in \mathbb{L}_{1}(P)$ with vector $e_{m}$ are linearizations of $P$. They are given by

$$
\lambda \mathcal{A}_{\mathcal{S}}+\mathcal{B}_{\mathcal{S}}=\left\{\begin{array}{l}
\lambda \mathcal{A}(1)+\mathcal{B}(1) \text { when } \mathcal{S} \in\{\text { Hermitian, symmetric }\} \\
\lambda \mathcal{A}(-1)+\mathcal{B}(-1) \text { when } \mathcal{S} \in\{\star \text {-even, } \star \star \text {-odd }\}
\end{array}\right.
$$

where

and

$$
\mathcal{A}(\varepsilon)=\left[\begin{array}{cccc}
0 & \cdots & 0 & \varepsilon^{m-1} A_{m} \\
\vdots & & . & \varepsilon^{m-2} A_{m-1} \\
\vdots & . \cdot & . & \vdots \\
\varepsilon^{0} A_{m} & \varepsilon^{0} A_{m-1} & \cdots & \varepsilon^{0} A_{1}
\end{array}\right]
$$

$$
\mathcal{B}(\varepsilon)=-\left[\begin{array}{ccccc}
0 & \ldots & 0 & \varepsilon^{m-1} A_{m} & 0 \\
\vdots & . & \varepsilon^{m-2} A_{m} & \varepsilon^{m-2} A_{m-1} & \vdots \\
0 & . & . & \vdots & \vdots \\
\varepsilon A_{m} & \varepsilon A_{m-1} & \cdots & \varepsilon A_{2} & 0 \\
0 & \cdots & \ldots & 0 & -A_{0}
\end{array}\right] .
$$

Note that for $\star$-(anti)palindromic $P(\lambda)$, we have $0 \notin \Lambda(P)$ since $\infty \notin \Lambda(P)$. When $m=2 k+1$ is odd, $v=e_{k+1}$ satisfies $v=F_{m} v=F_{m} \bar{v}$ and $0, \infty$ are the only roots of the v-polynomial. The corresponding $\star-\left(\right.$ anti)palindromic pencils in $\mathbb{L}_{1}(P)$ are given by

$$
\lambda \mathcal{A}_{\mathcal{S}}+\mathcal{B}_{\mathcal{S}}= \begin{cases}\lambda \mathcal{A}^{\text {odd }}+\left(\mathcal{A}^{\text {odd }}\right)^{\star} & \text { when } \mathcal{S}=\star \text {-palindromic with } m=2 k+1 \\ \lambda \mathcal{A}^{\text {odd }}-\left(\mathcal{A}^{\text {odd }}\right)^{\star} & \text { when } \mathcal{S}=\star \text {-antipalindromic with } m=2 k+1\end{cases}
$$

where

$$
\mathcal{A}^{\text {odd }}=\left[\begin{array}{ll}
\mathcal{A}_{11}^{\text {odd }} & \mathcal{A}_{12}^{\text {odd }} \\
\mathcal{A}_{21}^{\text {odd }} & \mathcal{A}_{22}^{\text {odd }}
\end{array}\right],
$$

with $\mathcal{A}_{11}^{\text {odd }}=\left(\mathcal{A}_{22}^{\text {odd }}\right)^{T}=0_{n k \times n(k+1)}$ and

$$
\mathcal{A}_{12}^{\text {odd }}=\left[\begin{array}{cccc}
-A_{m}^{\star} & 0 & \ldots & 0 \\
-A_{m-1}^{\star} & \ddots & & \vdots \\
\vdots & \ddots & \ddots & 0 \\
-A_{k+2}^{\star} & \ldots & -A_{m-1}^{\star} & -A_{m}^{\star}
\end{array}\right], \quad \mathcal{A}_{21}^{\text {odd }}=\left[\begin{array}{cccc}
A_{m} & A_{m-1} & \ldots & A_{k+1} \\
0 & \ddots & \ddots & \vdots \\
\vdots & \ddots & \ddots & A_{m-1} \\
0 & \ldots & 0 & A_{m}
\end{array}\right],
$$

are linearizations of $P(\lambda)$.

For $\star$-(anti)palindromic polynomials of even degree $m=2 k$, the simplest nonzero vector $v$ satisfying $F_{m} v=v$ when $\star=T$ or $F_{m} v=\bar{v}$ when $\star=*$ is of the form $v=\left[\begin{array}{lllllllll}0 & \ldots & 0 & z & z^{\star} & 0 & \ldots & 0\end{array}\right]^{T}$, where $z$ and $z^{\star}$ are in position $k$ and $k+1$, respectively. The corresponding $\star-\left(\right.$ anti)palindromic pencil in $\mathbb{L}_{1}(P)$ is a linearization of $P(\lambda)$ if $-z / z^{\star}$ is not an eigenvalue of $P$ and is given by

$$
\lambda \mathcal{A}_{\mathcal{S}}+\mathcal{B}_{\mathcal{S}}= \begin{cases}\lambda \mathcal{A}_{-}^{\text {even }}(z)+\left(\mathcal{A}_{-}^{\text {even }}(z)\right)^{\star} & \text { when } \mathcal{S}=\star \text {-palindromic with } m=2 k, \\ \lambda \mathcal{A}_{-}^{\text {even }}(z)-\left(\mathcal{A}_{-}^{\text {even }}(z)\right)^{\star} & \text { when } \mathcal{S}=\star \text {-antipalindromic with } m=2 k,\end{cases}
$$


where

$$
\mathcal{A}_{-}^{\text {even }}(z)=\left[\begin{array}{ll}
\mathcal{A}_{11}^{\text {even }}(z) & \mathcal{A}_{12}^{\text {even }}(z) \\
\mathcal{A}_{21}^{\text {even }}(z) & \mathcal{A}_{22}^{\text {even }}(z)
\end{array}\right]
$$

with

$$
\begin{aligned}
& \mathcal{A}_{11}^{\text {even }}(z)=z\left[\begin{array}{cccc}
0 & 0 & \ldots & 0 \\
\vdots & \vdots & & \vdots \\
0 & 0 & \ldots & 0 \\
A_{m} & A_{m-1} & \ldots & A_{k+1}
\end{array}\right], \quad \mathcal{A}_{22}^{\text {even }}(z)=z\left[\begin{array}{cccc}
A_{k+1} & 0 & \ldots & 0 \\
\vdots & \vdots & & \vdots \\
A_{m-1} & 0 & \ldots & 0 \\
A_{m} & 0 & \ldots & 0
\end{array}\right] \text {, } \\
& \mathcal{A}_{12}^{\text {even }}(z)=-\left[\begin{array}{cccccc}
z^{\star} A_{0} & z A_{0} & 0 & \cdots & \cdots & 0 \\
z^{\star} A_{1} & z^{\star} A_{0}+z A_{1} & \ddots & \ddots & & \vdots \\
\vdots & \vdots & \ddots & \ddots & \ddots & \vdots \\
\vdots & & \ddots & \ddots & \ddots & 0 \\
z^{\star} A_{k-2} & z^{\star} A_{k-2}+z A_{k-1} & \cdots & z^{\star} A_{1}+z A_{2} & z^{\star} A_{0}+z A_{1} & z A_{0} \\
-z A_{k}+z^{\star} A_{k-1} & z^{\star} A_{k-2} & & \cdots & z^{\star} A_{1} & z^{\star} A_{0}
\end{array}\right] \text {, } \\
& \mathcal{A}_{21}^{\text {even }}(z)=\left[\begin{array}{cccccc}
z^{\star} A_{m} & z A_{m}+z^{\star} A_{m-1} & z A_{m-1}+z^{\star} A_{m-2} & \ldots & \ldots & z A_{k+2}+z^{\star} A_{k+1} \\
0 & \ddots & \ddots & \ddots & \vdots \\
\vdots & \ddots & \ddots & \ddots & \\
\vdots & & \ddots & \ddots & \ddots & z A_{m-1}+z^{\star} A_{m-2} \\
\vdots & & & \ddots & z^{\star} A_{m} & z A_{m}+z^{\star} A_{m-1} \\
0 & \ldots & \ldots & \ldots & 0 & z^{\star} A_{m}
\end{array}\right] \text {. }
\end{aligned}
$$

Note that when $\star=*$, we can always pick a $z \in \mathbb{F}$ such that $-z / z^{\star} \notin \Lambda(P)$. But when $\star=T,-z / z^{\star}=-1$ so if $-1 \in \Lambda(P)$ the corresponding $\star$-(anti)palindromic pencil in $\mathbb{L}_{1}(P)$ is not a linearization of $P(\lambda)$. In fact it is shown in [15] that some $T$-(anti)palindromic matrix polynomials of even degree do not have $T$-(anti)palindromic linearizations. Instead, we allow a linearization with "anti" structure: palindromic becomes antipalindromic and vice versa. For this, let $v=\left[\begin{array}{llllllll}0 & \ldots & 0 & 1 & -1 & 0 & \ldots & 0\end{array}\right]^{T}$ satisfying $v=-F_{m} v$. If $P(\lambda)$ is $T$-palindromic then there is a unique $T$-antipalindromic pencil in $\mathbb{L}_{1}(P)$ with vector $v$. Similarly if $P(\lambda)$ is $T$-antipalindromic then there is unique $T$-palindromic pencil in $\mathbb{L}_{1}(P)$ with vector $v$. Such pencils are linearizations of $P$ if $1 \notin \Lambda(P)$ and are given by

$$
\lambda \mathcal{A}_{\mathcal{S}}+\mathcal{B}_{\mathcal{S}}= \begin{cases}\lambda \mathcal{A}_{+}^{\text {even }}-\left(\mathcal{A}_{+}^{\text {even }}\right)^{T} & \text { when } \mathcal{S}=T \text {-palindromic with } m=2 k, \\ \lambda \mathcal{A}_{+}^{\text {even }}+\left(\mathcal{A}_{+}^{\text {even }}\right)^{T} & \text { when } \mathcal{S}=T \text {-antipalindromic when } m=2 k,\end{cases}
$$

where $\mathcal{A}_{+}^{\text {even }}(z)$ has a block structure similar to that of $\mathcal{A}_{-}^{\text {even }}(z)$ in $(7)$ with $z$ replaced by 1 and $z^{\star}$ replaced by -1 . In particular, when $m=2$,

$$
\mathcal{A}_{+}^{\text {even }}=\left[\begin{array}{cc}
A_{2} & A_{1}+A_{0} \\
-A_{2} & A_{2}
\end{array}\right] \text {. }
$$

The next result, useful later, shows that the linearizations (4)-(8) share a property.

Lemma 2.1 Let $\mathcal{S} \in \mathbb{S}$ and $P(\lambda) \in \mathcal{P}_{\mathcal{S}}\left(\mathbb{F}^{n}\right)$ with nonsingular leading coefficient. If $\lambda \mathcal{A}_{\mathcal{S}}+\mathcal{B}_{\mathcal{S}}$ is a structured linearization of $P(\lambda)$ as in (4)-(8) then $\mathcal{C}=-\mathcal{A}_{\mathcal{S}}^{-1} \mathcal{B}_{\mathcal{S}}$, where $\mathcal{C}$ is the companion form of $P(\lambda)$ given in (3). 
Proof. Some easy calculations show that $-\mathcal{A}_{\mathcal{S}} \mathcal{C}=\mathcal{B}_{\mathcal{S}}$.

Hence, with the exception of $T$-(anti)palindromic $P(\lambda)$ of even degree with both -1 and 1 as eigenvalues, the companion form of $P(\lambda)$ can be factorized as $\mathcal{C}=-\mathcal{A}_{\mathcal{S}}^{-1} \mathcal{B}_{\mathcal{S}}$, where $\lambda \mathcal{A}_{\mathcal{S}}+\mathcal{B}_{\mathcal{S}}=\mathcal{A}_{\mathcal{S}}(\lambda I-\mathcal{C})$ is a structured linearization of $P(\lambda)$.

\subsection{Standard triples}

Recall that $(U, T)$ is an $(m, n)$-standard pair over $\mathbb{F}$ if $T \in \mathbb{F}^{m n \times m n}$ and $U \in \mathbb{F}^{n \times m n}$ are such that

$$
Q=Q(U, T):=\left[\begin{array}{c}
U T^{m-1} \\
\vdots \\
U T \\
U
\end{array}\right]
$$

is nonsingular [12, Def. 2.1]. The triple $(U, T, V)$ forms an $(m, n)$-standard triple over $\mathbb{F}$ if $(U, T)$ is an $(m, n)$-standard pair over $\mathbb{F}$ and $V \in \mathbb{F}^{m n \times n}$ is such that $U T^{m-1} V$ is nonsingular and, if $m \geq 2$,

$$
U T^{j} V=0, \quad j=0: m-2,
$$

or equivalently,

$$
Q V=e_{1} \otimes N
$$

for some nonsingular $n \times n$ matrix $N$, where $e_{1}$ is the first column of the $m \times m$ identity matrix [12, Def. 2.3]. Note that the definitions of standard pairs and triples make no reference to matrix polynomials.

An $(m, n)$-standard pair $(U, T)$ over $\mathbb{F}$ is a standard pair for $P(\lambda)=\sum_{j=0}^{m} \lambda^{j} A_{j}$ if

$$
A_{m} U T^{m}+A_{m-1} U T^{m-1}+\cdots+A_{1} U T+A_{0} U=0
$$

[6, p. 46]. A standard triple $(U, T, V)$ is a standard triple for $P(\lambda)$ if $(12)$ holds and $A_{m}=\left(U T^{m-1} V\right)^{-1}$. Any $P(\lambda) \in \mathcal{P}\left(\mathbb{F}^{n}\right)$ with nonsingular leading coefficient admits a standard triple. For example, it is easy to check that

$$
\left(e_{m}^{T} \otimes I_{n}, \mathcal{C}, e_{1} \otimes A_{m}^{-1}\right)
$$

with $\mathcal{C}$ as in (3) is a standard triple for $P(\lambda)$.

Let $U_{i} \in \mathbb{F}^{n \times m n}, T_{i} \in \mathbb{F}^{m n \times m n}$ and $V_{i} \in \mathbb{F}^{m n \times n}, i=1,2$. Then $\left(U_{1}, T_{1}, V_{1}\right)$ is similar to $\left(U_{2}, T_{2}, V_{2}\right)$ if there exists a nonsingular $G \in \mathbb{F}^{m n \times m n}$ such that

$$
U_{2}=U_{1} G, \quad T_{2}=G^{-1} T_{1} G, \quad V_{2}=G^{-1} V_{1} .
$$

Moreover if $\left(U_{1}, T_{1}, V_{1}\right)$ is a standard triple so is $\left(U_{2}, T_{2}, V_{2}\right)$ [5, Prop. 12.1.3]. Note that if $(U, T, V)$ is a standard triple for $P(\lambda)$ then

$$
\left(e_{m}^{T} \otimes I_{n}\right) Q=U, \quad Q^{-1} \mathcal{C} Q=T, \quad e_{1} \otimes A_{m}^{-1}=Q V,
$$

with $Q$ as in (9). Hence any standard triple $(U, T, V)$ for $P(\lambda)$ is similar to $\left(e_{m}^{T} \otimes\right.$ $\left.I_{n}, \mathcal{C}, e_{1} \otimes A_{m}^{-1}\right)$. Note that because $T$ is similar to $\mathcal{C}, \lambda I-T$ is a linearization of $P(\lambda)$ and $\Lambda(P)=\Lambda(T)$. The following result [5, Thm. 12.1.4] will be useful. 
Table 2: Definition of $u_{\mathcal{S}}(T), t_{\mathcal{S}}(T), v_{\mathcal{S}}(T)$ for some $T \in \mathbb{F}^{m n \times m n}$ satisfying assumption (b), where $\alpha$ is some scalar in $\mathbb{F}$ such that $\alpha^{\star} \alpha=1$ and $-\alpha \notin \Lambda(T)$.

\begin{tabular}{l|c|c|c} 
Structure $\mathcal{S}$ & $u_{\mathcal{S}}(T)$ & $t_{\mathcal{S}}(T)$ & $v_{\mathcal{S}}(T)$ \\
\hline Hermitian/symmetric & $I$ & $T^{\star}$ & $I$ \\
$\star$-even & $-I$ & $-T^{\star}$ & $I$ \\
$\star$-odd & $I$ & $-T^{\star}$ & $I$ \\
$\star$-palindromic, $m=2 k+1$ & $-T^{\star(k-1)}$ & $T^{-\star}$ & $T^{\star k}$ \\
$\star$-palindromic, $m=2 k$ & $-T^{\star(k-1)}\left(I+\alpha T^{\star}\right)^{-1}$ & $T^{-\star}$ & $\left(I+\alpha T^{\star}\right) T^{\star(k-1)}$ \\
$\star$-antipalindromic, $m=2 k+1$ & $T^{\star(k-1)}$ & $T^{-\star}$ & $T^{\star k}$ \\
$\star$-antipalindromicm $m=2 k$ & $T^{\star(k-1)}\left(I+\alpha T^{\star}\right)^{-1}$ & $T^{-\star}$ & $\left(I+\alpha T^{\star}\right) T^{\star(k-1)}$
\end{tabular}

Lemma 2.2 Let $U \in \mathbb{F}^{n \times m n}, T \in \mathbb{F}^{m n \times m n}, V \in \mathbb{F}^{m n \times n}$ and let $P(\lambda) \in \mathcal{P}\left(\mathbb{F}^{n}\right)$ be of degree $m$ with nonsingular leading coefficient. Then $(U, T, V)$ is a standard triple for $P(\lambda)$ if and only if $P(\lambda)^{-1}=U(\lambda I-T)^{-1} V$ for $\lambda \in \mathbb{C} \backslash \Lambda(P)$.

A Jordan triple $(X, J, Y)$ over $\mathbb{F}$ for $P(\lambda)$ is a standard triple for $P(\lambda)$ for which the matrix $J$ is in Jordan form or real Jordan form if $\mathbb{F}=\mathbb{R}$. By (12) and [6, Prop. 2.1], we have that $\sum_{j=0}^{m} A_{j} X J^{j}=0$ and $\sum_{j=0}^{m} J^{j} Y A_{j}=0$. The columns of $X$ and $Y^{*}$ determine right and left eigenvectors and generalized eigenvectors of $P(\lambda)$. The matrix $J$ is the Jordan form of the companion form $\mathcal{C}$ of $P(\lambda)$.

\section{3. $\mathcal{S}$-structured standard triples}

We now consider standard triples in the context of structured matrix polynomials. We start by listing two assumptions used in our analysis. Let $\mathcal{S} \in \mathbb{S}, P(\lambda) \in \mathcal{P}_{\mathcal{S}}\left(\mathbb{F}^{n}\right)$ have degree $m$ with nonsingular leading coefficient and let $T \in \mathbb{F}^{m n \times m n}$.

Assumption (a): if $\mathcal{S} \in\{T$-palindromic, $T$-antipalindromic $\}$ and $P(\lambda)$ has degree $m=2 k$ then either $-1 \notin \Lambda(P)$ or $1 \notin \Lambda(P)$.

Assumption (b): if $\mathcal{S} \in\{T$-palindromic, $T$-antipalindromic $\}$ and $m=2 k$ then either $-1 \notin \Lambda(T)$ or $1 \notin \Lambda(T)$.

These two assumptions are equivalent when $\lambda I-T$ is a linearization of $P(\lambda)$. For some $T$ satisfying assumption (b) we define $u_{\mathcal{S}}(T), t_{\mathcal{S}}(T), v_{\mathcal{S}}(T)$ as in Table 2 . Note that assumption (b) ensures the existence of $\alpha \in \mathbb{F}$ such that $\alpha^{\star} \alpha=1$ and $-\alpha \notin \Lambda(T)$. Also, for $\star$-(anti)palindromic structures, the eigenvalues of $T$ comes in pairs $\left(\lambda, \lambda^{-\star}\right)$. Hence $0 \notin \Lambda(T)$ since $\infty \notin \Lambda(T)$ and $T^{-\star}$ is well defined.

Before stating our main result in Theorem 3.5, we provide a few lemmas and introduce the notion of $\mathcal{S}$-structured standard triple. The first lemma of this section extends to all structures in $\mathbb{S}$ a result in $[11$, Cor. 14.2.1] for Hermitian structure.

Lemma 3.1 Let $(U, T, V)$ be an $(m, n)$-standard triple for $P(\lambda) \in \mathcal{P}\left(\mathbb{F}^{n}\right)$ with nonsingular leading coefficient and let $\mathcal{S} \in \mathbb{S}$. Assume that $T$ satisfies assumption $(b)$. Then $P(\lambda)$ has structure $\mathcal{S}$ if and only if $\left(V^{\star} u_{\mathcal{S}}(T), t_{\mathcal{S}}(T), v_{\mathcal{S}}(T) U^{\star}\right)$ is a standard triple for $P(\lambda)$. 
Proof. The proof for $\mathcal{S} \in\{$ Hermitian, symmetric, $\star$-even, $\star$-odd $\}$ is easy to obtain using the resolvent form for $P(\lambda)$ given in Lemma 2.2 and the definition of the structures in Table 1. See also [11, Cor. 14.2.1] for the Hermitian structure.

Now suppose that $P(\lambda)$ is $\star$-palindromic. Since any standard triple for $P(\lambda)$ is similar to $\left(e_{m}^{T} \otimes I_{n}, \mathcal{C}, e_{1} \otimes A_{m}^{-1}\right)=:(\widetilde{U}, \mathcal{C}, \widetilde{V})$, it suffices to show that this standard triple is similar to $\left(\widetilde{V}^{\star} u_{\mathcal{S}}(\mathcal{C}), \mathcal{C}^{-\star}, v_{\mathcal{S}}(\mathcal{C}) \widetilde{U}^{\star}\right)$. We need to consider three cases:

(i) $m=2 k+1$. The pencil $\lambda \mathcal{A}^{\text {odd }}+\left(\mathcal{A}^{\text {odd }}\right)^{\star}$ with $\mathcal{A}^{\text {odd }}$ as in (6) is a linearization of $P(\lambda)$. By Lemma $2.1, \mathcal{C}=-\left(\mathcal{A}^{\text {odd }}\right)^{-1}\left(\mathcal{A}^{\text {odd }}\right)^{\star}$. So if we let $G^{-1}=\mathcal{A}^{\text {odd }}$ then

$$
\widetilde{V}^{\star} u_{\mathcal{S}}(\mathcal{C})=-\left(e_{1} \otimes A_{m}^{-1}\right)^{\star}\left(\mathcal{C}^{\star}\right)^{k-1}=\widetilde{U} G, \quad G^{-1} \mathcal{C} G=\mathcal{C}^{-\star}=t_{\mathcal{S}}(\mathcal{C}),
$$

and

$$
G^{-1} \widetilde{V}=G^{-1}\left(e_{1} \otimes A_{m}^{-1}\right)=e_{2 k} \otimes I=\left(\mathcal{C}^{\star}\right)^{k}\left(e_{m} \otimes I\right)=v_{\mathcal{S}}(\mathcal{C}) \widetilde{U}^{\star} .
$$

(ii) $m=2 k, \star=T$ and $-1 \in \Lambda(T)$. From assumption (b) it follows that $1 \notin \Lambda(T)$ so we can take $\alpha=-1$ in the definition of $u_{\mathcal{S}}$ and $v_{\mathcal{S}}$. The pencil $\lambda \mathcal{A}_{+}^{\text {even }}-\left(\mathcal{A}_{+}^{\text {even }}\right)^{\star}$ with $\mathcal{A}_{+}^{\text {even }}$ as in (8) is a linearization of $P(\lambda)$. By Lemma $2.1, \mathcal{C}=-\left(\mathcal{A}_{+}^{\text {even }}\right)^{-1}\left(\mathcal{A}_{+}^{\text {even }}\right)^{\star}$. If we let $G^{-1}=\mathcal{A}_{+}^{\text {even }}$ then as in (i), $G^{-1} \mathcal{C} G=t_{\mathcal{S}}(\mathcal{C})$. Also,

$$
v_{\mathcal{S}}(\mathcal{C}) \widetilde{U}^{T}=\left(I-\mathcal{C}^{T}\right) \mathcal{C}^{T(k-1)}\left(e_{m} \otimes I_{n}\right)=e_{k+1} \otimes I-e_{k} \otimes I=G^{-1}\left(e_{1} \otimes I\right) A_{m}^{-1}=G^{-1} \widetilde{V} .
$$

From $\widetilde{V}=G v_{\mathcal{S}}(\mathcal{C}) \widetilde{U}^{T}$ it follows that $\widetilde{V}^{T}=\widetilde{U} \mathcal{C}^{(k-1)}(I-\mathcal{C}) G^{T}$, so that

$$
\begin{aligned}
\widetilde{V}^{T} u_{\mathcal{S}}(\mathcal{C}) & =-\widetilde{U} \mathcal{C}^{(k-1)}(I-\mathcal{C}) G^{T} \mathcal{C}^{T(k-1)}\left(I-\mathcal{C}^{T}\right)^{-1} \\
& =-\widetilde{U} \mathcal{C}^{(k-1)}(I-\mathcal{C}) \mathcal{C}^{(1-k)} G^{T}\left(I-\mathcal{C}^{T}\right)^{-1} \\
& =\widetilde{U} G^{-1}\left(I-\mathcal{C}^{T}\right)\left(I-\mathcal{C}^{T}\right)^{-1}=\widetilde{U} G^{-1}
\end{aligned}
$$

where we used $G^{T} \mathcal{C}^{T(k-1)} G^{-T}=\mathcal{C}^{-(k-1)}$ and $\mathcal{C} G^{T}=G$.

(iii) $m=2 k, \star=*, T$ and $-1 \notin \Lambda(T)$. In this case Lemma 2.1 says that $\mathcal{C}=$ $-\left(\mathcal{A}_{-}^{\text {even }}(z)\right)^{-1}\left(\mathcal{A}_{-}^{\text {even }}(z)\right)^{\star}$ with $\mathcal{A}_{-}^{\text {even }}(z)$ as in $(7)$. The proof is similar to that in (ii) with $\alpha=z / z^{\star}$.

Conversely, suppose that $(U, T, V)$ and $\left(V^{\star} u_{\mathcal{S}}(T), T^{-\star}, v_{\mathcal{S}}(T) U^{\star}\right)$ are standard triples for $P(\lambda)$. Assumption (b) guarantees the existence of $\alpha \in \mathbb{F}$ such that $\alpha^{\star} \alpha=1$ and $I+\alpha T^{\star}$ is nonsingular. Hence $u_{\mathcal{S}}$ and $v_{\mathcal{S}}$ are well defined. Using the resolvent form for $P(\lambda), P(\lambda)^{-1}=U(\lambda I-T)^{-1} V$, we obtain

$$
\lambda^{-m}\left(P\left(\lambda^{-\star}\right)\right)^{-\star}=\lambda^{-m}\left(U\left(\lambda^{-\star} I-T\right)^{-1} V\right)^{\star}=\lambda^{1-m} V^{\star}\left(I-\lambda T^{\star}\right)^{-1} U^{\star} .
$$

If $\left\|\lambda T^{\star}\right\|<1$ for some subordinate matrix norm $\|\cdot\|$ then

$$
\left(I-\lambda T^{\star}\right)^{-1}=I+\lambda T^{\star}+\lambda^{2} T^{\star 2}+\cdots .
$$

Using (14) and the fact that $V^{\star} T^{\star j} U^{\star}=0, j=0: m-2$ (see (10)), we obtain

$$
\begin{aligned}
\lambda^{-m}\left(P\left(\lambda^{-\star}\right)\right)^{-\star} & =V^{\star} T^{\star(m-1)}\left(I+\lambda T^{\star}+\lambda^{2} T^{\star 2}+\cdots\right) U^{\star} \\
& =V^{\star} T^{\star(k-1)}\left(I-\lambda T^{\star}\right)^{-1} T^{\star(m-k)} U^{\star} \\
& =-V^{\star} T^{\star(k-1)}\left(\lambda I-T^{-\star}\right)^{-1} T^{\star(m-k-1)} U^{\star} \\
& \quad 8
\end{aligned}
$$


for all $|\lambda|<\left\|T^{\star}\right\|^{-1}$. When $m=2 k+1$, (15) reads $\lambda^{-m}\left(P\left(\lambda^{-\star}\right)\right)^{-\star}=V^{\star} u_{\mathcal{S}}(T)(\lambda I-$ $\left.T^{-\star}\right)^{-1} v_{\mathcal{S}}(T) U^{\star}=P(\lambda)^{-1}$. Note that $\left(\lambda I-T^{-\star}\right)^{-1}$ commutes with $T^{\star k-1},(I+$ $\left.\alpha T^{\star}\right)$ and $\left(I+\alpha T^{\star}\right)^{-1}$ so when $m=2 k,(15)$ can be rewritten as $\lambda^{-m}\left(P\left(\lambda^{-\star}\right)\right)^{-\star}=$ $V^{\star} u_{\mathcal{S}}(T)\left(\lambda I-T^{-\star}\right)^{-1} v_{\mathcal{S}}(T) U^{\star}=P(\lambda)^{-1}$ for all $|\lambda|<\|T\|^{-1}$. Since $\lambda^{-m}\left(P\left(\lambda^{-\star}\right)\right)^{-\star}=$ $P(\lambda)^{-1}$ holds for many values of $\lambda, P(\lambda)=\lambda^{m} P^{\star}\left(\lambda^{-1}\right)$ for all $\lambda$, that is, $P(\lambda)$ is $\star-$ palindromic.

The results for the $\star$-antipalindromic structure are proved in a similar way.

In the proof of Lemma 3.1 we use the fact that if $(U, T, V)$ is a standard triple for a structured $P(\lambda)$ then there exists a nonsingular $S$ such that

$$
U S=V^{\star} u_{\mathcal{S}}(T), \quad S^{-1} T S=t_{\mathcal{S}}(T), \quad S^{-1} V=v_{\mathcal{S}}(T) U^{\star} .
$$

These relations imply certain properties of $S$, which we use in our definition of $\mathcal{S}$ structured standard triples.

Definition $3.2(\mathcal{S}$-structured standard triple) Let $\mathcal{S} \in \mathbb{S}$. An $(m, n)$-standard triple $(U, T, V)$ with $T$ satisfying assumption $(b)$ is said to be $\mathcal{S}$-structured if $V=S v_{\mathcal{S}}(T) U^{\star}$ for some nonsingular $S \in \mathbb{F}^{m n \times m n}$ having the following properties:

- $S=S^{\star}, T S=(T S)^{\star}$ when $\mathcal{S} \in\{$ Hermitian, symmetric $\}$,

- $S=-S^{\star}, T S=(T S)^{\star}$ when $\mathcal{S}=\star$-even,

- $S=S^{\star}, T S=-(T S)^{\star}$ when $\mathcal{S}=\star-$ odd,

- $T S^{\star}=-S$ when $\mathcal{S}=\star$-palindromic and $m=2 k+1$ or $T S^{\star}=-\alpha S$ when $\mathcal{S}=$ $\star$-palindromic and $m=2 k$,

- $T S^{\star}=S$ when $\mathcal{S}=\star$-antipalindromic and $m=2 k+1$ or $T S^{\star}=\alpha S$ when $\mathcal{S}=$ $\star$-antipalindromic and $m=2 k$,

for some $\alpha \in \mathbb{F}$ such that $\alpha^{\star} \alpha=1$ and $-\alpha \notin \Lambda(T)$.

We refer to the matrix $S$ in Definition 3.2 as the $S$-matrix of the $\mathcal{S}$-structured standard triple $(U, T, V)$. We point out that Hermitian and symmetric structured standard triples are called self-adjoint standard triples in the literature (see for example [5, p. 244]). For $\star$-(anti)palindromic structures, the matrix $T$ in Definition 3.2 is $S^{-1}$-unitary, that is, $T^{\star} S^{-1} T=S^{-1}$, and with additional constraints on T's structure, Lancaster, Prells and Rodman refer to $(U, T, V)$ as a unitary standard triple [8, Def. 4]. Hence a unitary standard triple is $\mathcal{S}$-structured but the converse is not true in general.

Our definition of $\mathcal{S}$-structured standard triples is justified by the next lemma.

Lemma 3.3 Let $\mathcal{S} \in \mathbb{S}$. An $(m, n)$-standard triple $(U, T, V)$ with $T$ satisfying assumption (b) is $\mathcal{S}$-structured if and only if it is similar to $\left(V^{\star} u_{\mathcal{S}}(T), t_{\mathcal{S}}(T), v_{\mathcal{S}}(T) U^{\star}\right)$.

Proof. The proof of this lemma appears in [12, Thm. 3.4] for the symmetric structure and the proof there extends easily to structures $\mathcal{S} \in\{$ Hermitian, $\star$-even, $\star$-odd $\}$. 
Suppose $\mathcal{S}=\star$-palindromic (the proof for $\star$-antipalindromic structure is similar and so we omit it). If $m=2 k+1$ and $(U, T, V)$ is $\mathcal{S}$-structured, then there exists $S$ nonsingular such that $T S^{\star}=-S$ and $V=S T^{\star k} U^{\star}$. Hence $S^{-1} T S=T^{-\star}$ and

$$
U S=V^{\star} S^{-\star} T^{-k} S=-V^{\star} S^{-\star} T^{-(k-1)} S^{\star}=-V^{\star} T^{\star(k-1)} .
$$

Hence $(U, T, V)$ is similar to $\left(-V^{\star} T^{\star(k-1)}, T^{-\star}, T^{\star(m-k-1)} U^{\star}\right)$.

When $m=2 k, T S^{\star}=-\alpha S$ and $V=S\left(I+\alpha T^{\star}\right) T^{\star(k-1)} U^{\star}$ for some $\alpha \in \mathbb{F}$ such that $\alpha^{\star} \alpha=1$ and $-\alpha \notin \Lambda(T)$. The first equality implies that $S^{-1} T S=T^{-\star}$ while the first and second equality yield

$$
\begin{aligned}
U S & =V^{\star} S^{-\star} T^{-(k-1)}\left(I+\alpha^{\star} T\right)^{-1} S \\
& =V^{\star} T^{\star(k-1)} S^{-\star}\left(I+\alpha^{\star} T\right)^{-1} S \\
& =-V^{\star} T^{\star(k-1)}\left(I+\alpha T^{\star}\right)^{-1}=V^{\star} u_{\mathcal{S}}(T) .
\end{aligned}
$$

Conversely, if $(U, T, V)$ is similar to $\left(V^{\star} u_{\mathcal{S}}(T), T^{-\star}, v_{\mathcal{S}}(T) U^{\star}\right)$ then there exists $S$ nonsingular such that (16) holds with $t_{\mathcal{S}}(T)=T^{-\star}$. It remains to show that $T S^{\star}=-S$ when $m=2 k+1$ and $T S^{\star}=-\alpha S$ when $m=2 k$. When $m=2 k+1$, the first and last equalities in (16) imply that $V=-S T^{\star k} S^{-\star} T^{(k-1)} V$ and since $V$ has full rank, $-S T^{\star k} S^{-\star} T^{(k-1)}=I$, which on using the second equality in (16) yields $T S^{\star}=-S$. When $m=2 k$, the first and last equalities in (16) and the fact that $V$ has full rank imply that

$$
\begin{array}{ll} 
& -S T^{\star(k-1)}\left(I+\alpha T^{\star}\right) S^{-\star}\left(I+\alpha^{\star} T\right)^{-1} T^{(k-1)}=I \\
\Longleftrightarrow \quad & -T^{-(k-1)}\left(I+\alpha T^{-1}\right) S S^{-\star}\left(I+\alpha^{\star} T\right)^{-1} T^{(k-1)}=I \\
\Longleftrightarrow \quad & -T^{-(k-1)} S S^{-\star}\left(\alpha T^{-1}\right) T^{(k-1)}=I \\
\Longleftrightarrow & -\alpha S S^{-\star}=T^{(k-1)} T^{-(k-2)}=T .
\end{array}
$$

The next lemma shows that any standard triple that is similar to an $\mathcal{S}$-structured standard triple is itself $\mathcal{S}$-structured.

Lemma 3.4 Let $(U, T, V)$ be a standard triple similar to $\left(U_{1}, T_{1}, V_{1}\right)$, that is, $\left(U_{1}, T_{1}, V_{1}\right)=$ $\left(U G, G^{-1} T G, G^{-1} V\right)$ for some nonsingular matrix $G$. Let $\mathcal{S} \in \mathbb{S}$ and assume $T$ satisfies assumption (b). If $(U, T, V)$ is $\mathcal{S}$-structured with matrix $S$ then $\left(U_{1}, T_{1}, V_{1}\right)$ is $\mathcal{S}$ structured with matrix $S_{1}=G^{-1} S G^{-\star}$.

Proof. It is easy to check that $V_{1}=S_{1} v_{\mathcal{S}}\left(T_{1}\right) U_{1}^{\star}$ and since the properties of $S$ are preserved by $\star$-congruence, $\left(U_{1}, T_{1}, V_{1}\right)$ is $\mathcal{S}$-structured with matrix $S_{1}$.

We can now state our main result, which is a direct consequence of Lemma 3.1, Lemma 3.3 and Lemma 3.4. It extends a result for Hermitian structure [5, Thm. 12.2.2] to all structures in $\mathbb{S}$.

Theorem 3.5 Let $\mathcal{S} \in \mathbb{S}$ and $P(\lambda) \in \mathcal{P}\left(\mathbb{F}^{n}\right)$ with nonsingular leading coefficient satisfying assumption $(a)$. Then $P(\lambda)$ has structure $\mathcal{S}$ if and only if $P(\lambda)$ admits an $\mathcal{S}$-structured standard triple, in which case every standard triple for $P(\lambda)$ is $\mathcal{S}$-structured. 
The structure of the matrix $S$ in an $\mathcal{S}$-structured standard triple is uniquely determined by the triple, as shown by the next result.

Proposition 3.6 Let $\mathcal{S} \in \mathbb{S}$ and $(U, T, V)$ be an $\mathcal{S}$-structured standard triple with matrix S. Then

$$
S=Q(U, T)^{-1} Q\left(V^{\star} u_{\mathcal{S}}(T), t_{\mathcal{S}}(T)\right)
$$

with $Q(U, T)$ as in $(9)$.

Proof. Using Definition 3.2 we check that $Q(U, T) S=Q\left(V^{\star} u_{\mathcal{S}}(T), t_{\mathcal{S}}(T)\right)$.

The matrix $S$ is also easy to construct when the matrix coefficients of $P(\lambda)$ are known.

Proposition 3.7 Let $\mathcal{S} \in \mathbb{S}$ and $P(\lambda) \in \mathcal{P}_{\mathcal{S}}\left(\mathbb{F}^{n}\right)$ of degree $m$ with nonsingular leading coefficient satisfying assumption $(a)$. If $(U, T)$ is a standard pair for $P(\lambda)$ then $\left(U, T, S v_{\mathcal{S}}(T) U^{\star}\right)$ is an $\mathcal{S}$-structured standard triple for $P(\lambda)$ with matrix $S$ given by

$$
S^{-1}= \begin{cases}Q^{T} \mathcal{A}_{+}^{\text {even }} Q & \text { if } P \text { is } T \text {-(anti) palindromic, } m=2 k \text { and }-1 \in \Lambda(P), \\ z^{-\star} Q^{\star} \mathcal{A}_{-}^{\text {even }}(z) Q & \text { if } P \text { is } \star \text {-(anti) palindromic, } m=2 k,-z / z^{\star} \notin \Lambda(P), \\ Q^{\star} \mathcal{A}_{\mathcal{S}} Q & \text { otherwise, }\end{cases}
$$

where $Q:=Q(U, T)$ is as in $(9)$, and $\mathcal{A}_{\mathcal{S}}, \mathcal{A}_{-}^{\text {even }}(z)$ and $\mathcal{A}_{+}^{\text {even }}$ are as in $(4)-(8)$.

Proof. We first show that the matrix $S$ in the proposition has the properties listed in Definition 3.2. Note that under assumption (a), $P(\lambda)$ has a structured linearization $\lambda A_{\mathcal{S}}+\mathcal{B}_{\mathcal{S}}$, which is one of (4)-(8). The pair $(Q, T)$ is a standard pair for $\lambda \mathcal{A}_{\mathcal{S}}+\mathcal{B}_{\mathcal{S}}$, and hence

$$
Q^{\star} \mathcal{A}_{\mathcal{S}} Q T=-Q^{\star} \mathcal{B}_{\mathcal{S}} Q \quad \Longleftrightarrow \quad Q^{\star} \mathcal{B}_{\mathcal{S}} Q=-z^{\star} S^{-1} T,
$$

where $z=1$ except when $\mathcal{A}_{\mathcal{S}}=\mathcal{A}_{-}^{\text {even }}(z)$, in which case $z$ is such that $-z / z^{\star} \notin \Lambda(P)$. Since $\star$-congruence preserves any structure in $\mathbb{S}$, the pencil

$$
Q^{\star}\left(\lambda \mathcal{A}_{\mathcal{S}}+\mathcal{B}_{\mathcal{S}}\right) Q=z^{\star}\left(\lambda S^{-1}-S^{-1} T\right)
$$

has the same structure as $\lambda \mathcal{A}_{\mathcal{S}}+\mathcal{B}_{\mathcal{S}}$, and hence $S$ satisfies the appropriate properties.

It remains to show that $V$ in (11) has the form $V=S v_{\mathcal{S}}(T) U^{\star}$. For $\mathcal{S} \in\{$ Hermitian, symmetric, $\star$-even, $\star$-odd $\}, v_{\mathcal{S}}(T)=I$. Since $U^{\star}=Q^{\star}\left(e_{m} \otimes I_{n}\right)$ and $S^{-1}=Q^{\star} \mathcal{A}_{\mathcal{S}} Q$, we find that

$$
S U^{\star}=Q^{-1} \mathcal{A}_{\mathcal{S}}^{-1} Q^{-\star} Q^{\star}\left(e_{m} \otimes I_{n}\right)=Q^{-1} \mathcal{A}_{\mathcal{S}}^{-1}\left(e_{m} \otimes I_{n}\right) .
$$

From the block structure of $\mathcal{A}_{\mathcal{S}}$ in (4) we see that $\mathcal{A}_{\mathcal{S}}\left(e_{1} \otimes I_{n}\right)=\left(e_{m} \otimes I\right) A_{m}$, or equivalently, $\mathcal{A}_{\mathcal{S}}^{-1}\left(e_{m} \otimes I\right)=\left(e_{1} \otimes I_{n}\right) A_{m}^{-1}$ since $\mathcal{A}_{\mathcal{S}}$ and $A_{m}$ are both nonsingular. Hence $S U^{\star}=Q^{-1}\left(e_{1} \otimes I_{n}\right) A_{m}^{-1}=V$.

When $P(\lambda)$ is $\star$-(anti)palindromic of odd degree then the definition of $V$ in (11), the expression for $S$ in the proposition and the structure of $\mathcal{A}_{\mathcal{S}}=\mathcal{A}^{\text {odd }}$ in (5) yield

$$
S^{-1} V=Q^{\star} \mathcal{A}_{\mathcal{S}} Q Q^{-1}\left(e_{1} \otimes I_{n}\right) A_{m}^{-1}=T^{\star k} U^{\star} .
$$

For a $\star$-palindromic $P$ of even degree, we have shown that $T S^{\star}=-\alpha S$, where $\alpha=$ $-z / z^{\star}$. From the definition of $Q$ in $(9), T^{\star(k-1)} U^{\star}=Q^{\star}\left(e_{k+1} \otimes I_{n}\right)$. Hence on using 
the definition of $V$ in (11), the expression for $S$ in the proposition and the structure of $\mathcal{A}_{\mathcal{S}}=\mathcal{A}_{-}^{\text {even }}(z)$ in $(7)$ we have that

$$
\begin{aligned}
Q S\left(I+\alpha T^{\star}\right) & =Q\left(S-S^{\star}\right) Q^{\star}\left(e_{k+1} \otimes I_{n}\right) \\
& =\left(z^{\star} \mathcal{A}_{-}^{\text {even }}(z)\right)^{-1}\left(e_{k+1} \otimes I_{n}\right)-z\left(\mathcal{A}_{-}^{\text {even }}(z)\right)^{-\star}\left(e_{k+1} \otimes I_{n}\right) .
\end{aligned}
$$

From the definition of $\mathcal{A}_{-}^{\text {even }}(z)$ in (7), we find that

$$
\begin{gathered}
\mathcal{A}_{-}^{\text {even }}(z)\left[\begin{array}{c}
z^{-1} A_{m}^{-1}-z^{m-1} P\left(z,-z^{\star}\right)^{-1} \\
(-z)^{m-2} z^{\star} P\left(z,-z^{\star}\right)^{-1} \\
(-z)^{m-3} z^{\star 2} P\left(z,-z^{\star}\right)^{-1} \\
\vdots \\
(-z)^{0} z^{\star(m-1)} P\left(z,-z^{\star}\right)^{-1}
\end{array}\right]=-\alpha^{-1} e_{k+1} \otimes I_{n}, \\
\left(\mathcal{A}_{-}^{\text {even }}(z)\right)^{\star}\left[\begin{array}{c}
-z^{m-1}\left(z^{\star}\right)^{0} P\left(z,-z^{\star}\right)^{-1} \\
(-z)^{m-2} z^{\star} P\left(z,-z^{\star}\right)^{-1} \\
(-z)^{m-3} z^{\star 2} P\left(z,-z^{\star}\right)^{-1} \\
\vdots \\
(-z)^{0} z^{\star(m-1)} P\left(z,-z^{\star}\right)^{-1}
\end{array}\right]=e_{k+1} \otimes I_{n},
\end{gathered}
$$

where $P\left(z,-z^{\star}\right)=\sum_{j=0}^{m} z^{j}\left(-z^{\star}\right)^{m-j} A_{j}$. Hence

$$
z^{\star}\left(\mathcal{A}_{-}^{\text {even }}(z)\right)^{-1}\left(e_{k+1} \otimes I_{n}\right)-z\left(\mathcal{A}_{-}^{\text {even }}(z)\right)^{-\star}\left(e_{k+1} \otimes I_{n}\right)=\left(e_{1} \otimes I_{n}\right) A_{m}^{-1},
$$

that is, $S\left(I+\alpha T^{\star}\right) T^{\star(k-1)} U^{\star}=Q^{-1}\left(e_{1} \otimes I_{n}\right) A_{m}^{-1}=V$. The proof for $\star$-antipalindromic $P$ is along the same lines.

It follows from Theorem 3.5 and Proposition 3.7 that if $P(\lambda)$ has structure $\mathcal{S}$ then the standard triple (13) is $\mathcal{S}$-structured with matrix $S=\mathcal{A}_{\mathcal{S}}^{-1}$ except when $\mathcal{A}_{\mathcal{S}}=\mathcal{A}_{-}^{\text {even }}(z)$, in which case $S=z^{\star}\left(\mathcal{A}_{-}^{\text {even }}(z)\right)^{-1}$.

\section{4. $\mathcal{S}$-structured Jordan triples}

We now explain how to obtain explicit expressions for the Jordan matrix and $S$-matrix of $\mathcal{S}$-structured Jordan triples $\left(X, J, S_{J} v_{\mathcal{S}}(J) X^{\star}\right)$ of $P(\lambda) \in \mathcal{P}_{\mathcal{S}}\left(\mathbb{F}^{n}\right)$. We note that the matrix $S_{J}$ displays the sign characteristic of $P(\lambda)$, whose definition we now give.

Let $\left(U, T, S_{T} v_{\mathcal{S}}(T) U^{\star}\right)$ be a standard triple for $P(\lambda) \in \mathcal{P}_{\mathcal{S}}\left(\mathbb{F}^{n}\right)$. The sign characteristic of $P(\lambda)$ is defined as the sign characteristic of the pair $\left(T, S_{T}^{-1}\right)$, which is a list of signs, with a sign $(+1$ or -1$)$ attached to each partial multiplicity of

- real eigenvalues of Hermitian or real symmetric matrix polynomials,

- purely imaginary eigenvalues of $*$-even, $*$-odd, real $T$-even and real $T$-odd matrix polynomials,

- eigenvalues with unit modulus of $*$-(anti)palindromic and real $T$-palindromic matrix polynomials. 
These signs can be read off the canonical decomposition of $\lambda S_{T}^{-1}-S_{T}^{-1} T$ via $\star$-congruence (see [5, Sec. 12.4] for Hermitian structure). Note that the definition of the sign characteristic for $P(\lambda)$ is independent of the choice of standard triple. Indeed if $\left(U_{i}, T_{i}, S_{T_{i}} v_{\mathcal{S}}\left(T_{i}\right) U_{i}^{\star}\right)$, $i=1,2$ are $\mathcal{S}$-structured standard triples for $P(\lambda)$, then by Lemma 3.4 there exists a nonsingular $G$ such that $T_{2}=G^{-1} T_{1} G$ and $S_{T_{2}}=G^{-1} S_{T_{1}} G^{-\star}$. Hence, $\lambda S_{T_{2}}^{-1}-S_{T_{2}}^{-1} T_{2}=$ $G^{\star}\left(\lambda S_{T_{1}}^{-1}-S_{T_{1}}^{-1} T_{1}\right) G$, that is, the pencils $\lambda S_{T_{i}}^{-1}-S_{T_{i}}^{-1} T_{i}, i=1,2$ are $\star$-congruent. They share the same canonical decomposition via $\star$-congruence and therefore the same sign characteristic.

We know that the triple $\left(\left(e_{m}^{T} \otimes I_{n}\right), \mathcal{C},\left(e_{1} \otimes A_{m}^{-1}\right)\right)$ is a standard triple for $P(\lambda)$ and by Theorem 3.5, it is $\mathcal{S}$-structured with $S$-matrix as in Proposition 3.7 with $Q=I_{m n}$. Hence, on using Lemma 2.1, we find that

$$
\lambda S_{\mathcal{C}}^{-1}-S_{\mathcal{C}}^{-1} \mathcal{C}=\lambda z^{-\star} \mathcal{A}_{\mathcal{S}}+z^{-\star} \mathcal{B}_{\mathcal{S}},
$$

where $\lambda \mathcal{A}_{\mathcal{S}}+\mathcal{B}_{\mathcal{S}}$ is a structured linearization of $P(\lambda)$ as in (4)-(8), and $z=1$ except when $\mathcal{A}_{\mathcal{S}}=\mathcal{A}_{-}^{\text {even }}(z)$, in which case $z \in \mathbb{F}$ is chosen such that $-z / z^{\star} \notin \Lambda(P)$. So what we need is a canonical decomposition of $\lambda \mathcal{A}_{\mathcal{S}}+\mathcal{B}_{\mathcal{S}}$ via $\star$-congruence,

$$
Z^{\star}\left(\lambda \mathcal{A}_{\mathcal{S}}+\mathcal{B}_{\mathcal{S}}\right) Z=\lambda\left(Z^{\star} \mathcal{A}_{\mathcal{S}} Z\right)-\left(Z^{\star} \mathcal{A}_{\mathcal{S}} Z\right)\left(Z^{-1} \mathcal{C} Z\right)=z^{\star}\left(\lambda S_{J}^{-1}-S_{J}^{-1} J\right),
$$

where $J=Z^{-1} \mathcal{C} Z$ is the Jordan form of $\mathcal{C}$. Fortunately, such decompositions are available in the literature for all the structures in $\mathbb{S}$. We use these canonical decompositions to provide explicit expressions for $J$ and $S_{J}$ in Appendix A. These expressions show that $S_{J}$ and $J$ have the same block structure and that we can read the sign characteristic of $P(\lambda)$ from certain diagonal blocks of $S_{J}$.

\section{Concluding remarks}

The results in this paper represent a first step towards the solution of the structured inverse polynomial eigenvalue problem: given a list of admissible elementary divisors for the structure, and possibly, corresponding right eigenvectors and generalized eigenvectors, construct a structured matrix polynomial having these elementary divisors and eigenvectors/generalized eigenvectors. Indeed, using the results in sections 3 and 4 we show in [1] how to construct an $\mathcal{S}$-structured $(2, n)$-Jordan triple $(X, J, Y)$ from a given list of $2 n$ prescribed eigenvalues and $n$ linearly independent eigenvectors and generalized eigenvectors, and use the fact that an $\mathcal{S}$-structured $(2, n)$-Jordan triple defines a unique structured quadratic $Q(\lambda)=\lambda^{2} A_{2}+\lambda A_{1}+A_{0} \in \mathcal{P}_{\mathcal{S}}\left(\mathbb{F}^{n}\right)$, where $A_{2}=\left(X J S v_{\mathcal{S}}(J) X^{\star}\right)^{-1}$,

$$
A_{1}=-A_{2} X J^{2} S v_{\mathcal{S}}(J) X^{\star} A_{2}, \quad A_{0}=-A_{2}\left(X J^{2} S v_{\mathcal{S}}(J) X^{\star} A_{1}+X J^{3} S v_{\mathcal{S}}(J) X^{\star} A_{2}\right),
$$

and $v_{\mathcal{S}}(\cdot)$ as in Table 2 .

Finally, we note that standard triples have been useful to describe structure preserving transformations (SPTs) for matrix polynomials, and in particular quadratic matrix polynomials [3]. We believe that the notion of $\mathcal{S}$-structured standard triples will further our understanding of SPTs for structured matrix polynomials. 


\section{Appendix A. Explicit expressions for $J$ and $S_{J}$}

Using the canonical decompositions of structured pencils via $\star$-congruences, we provide in this appendix an explicit expression for the Jordan matrix and $S$-matrix of $\mathcal{S}$ structured Jordan triples $\left(X, J, S_{J} v_{\mathcal{S}}(J) X^{\star}\right)$ of $P(\lambda) \in \mathcal{P}_{\mathcal{S}}\left(\mathbb{F}^{n}\right)$ for each $\mathcal{S} \in \mathbb{S}$. We assume that $P(\lambda)$ is of degree $m$ with nonsingular leading coefficient matrix. To facilitate the description of $J$ and $S_{J}$, we introduce the matrices $F_{1}=[1], G_{1}=[0]$, and for integers $k>1$

$$
E_{k}=\left[\begin{array}{r}
1^{.1} \\
(-1)^{k-1}
\end{array}\right]_{k \times k}=(-1)^{k-1} E_{k}^{T}, \quad F_{k}=\left[\begin{array}{ll}
. & 1 \\
1 &
\end{array}\right]_{k \times k} .
$$

We denote by

$$
J_{\ell_{k}}\left(\lambda_{k}\right)=\left[\begin{array}{cccc}
\lambda_{k} & 1 & & \\
& \lambda_{k} & \ddots & \\
& & \ddots & 1 \\
& & & \lambda_{k}
\end{array}\right] \in \mathbb{C}^{\ell_{k} \times \ell_{k}},
$$

the Jordan block of size $\ell_{k}$ associated with $\lambda_{k}$, and by

$$
K_{2 m_{k}}\left(\lambda_{k}, \bar{\lambda}_{k}\right)=K_{2 m_{k}}\left(\Lambda_{k}\right)=\left[\begin{array}{cccc}
\Lambda_{k} & I_{2} & & \\
& \Lambda_{k} & \ddots & \\
& & \ddots & I_{2} \\
& & & \Lambda_{k}
\end{array}\right] \in \mathbb{R}^{2 m_{k} \times 2 m_{k}}, \quad \Lambda_{k}=\left[\begin{array}{cc}
\alpha_{k} & \beta_{k} \\
-\beta_{k} & \alpha_{k}
\end{array}\right],
$$

the $2 m_{k} \times 2 m_{k}$ real Jordan block associated with the pair of complex conjugate eigenvalues $\left(\lambda_{k}, \bar{\lambda}_{k}\right)$, where $\lambda_{k}=\alpha_{k}+i \beta_{k}$ with $\alpha_{k}, \beta_{k} \in \mathbb{R}, \beta_{k} \neq 0$. We use the notation $\bigoplus_{j=1}^{r} F_{j}$ to denote the direct sum of the matrices $F_{1}, \ldots, F_{r}$.

Note that there are restrictions on the Jordan structure of $P$. For instance, a regular $n \times n$ matrix polynomial cannot have more than $n$ elementary divisors associated with the same eigenvalue [14]. Also, the elementary divisors have certain pairing, which depends on the structure $\mathcal{S} \in \mathbb{S}$ and the eigenvalue. Hence we describe for each $\mathcal{S} \in \mathbb{S}$ the elementary divisors arising from $P(\lambda) \in \mathcal{P}_{\mathcal{S}}\left(\mathbb{F}^{n}\right)$ and then provide an expression for $J$ and $S_{J}$.

\section{Appendix A.1. Hermitian structure}

Suppose $P(\lambda)$ is Hermitian with

- $r$ real elementary divisors $\left(\lambda-\lambda_{j}\right)^{\ell_{j}}, j=1: r$, and

- $s$ pairs of nonreal conjugate elementary divisors $\left(\lambda-\mu_{j}\right)^{m_{j}},\left(\lambda-\bar{\mu}_{j}\right)^{m_{j}}, j=1: s$

with $\ell_{j}, m_{j}$ such that $\sum_{j=1}^{r} \ell_{j}+2 \sum_{j=1}^{s} m_{j}=m n$. It follows from [9, Thm. 6.1] that

$$
J=\bigoplus_{j=1}^{r} J_{\ell_{j}}\left(\lambda_{j}\right) \oplus \bigoplus_{j=1}^{s}\left(J_{m_{j}}\left(\bar{\mu}_{j}\right) \oplus J_{m_{j}}\left(\mu_{j}\right)\right), \quad S_{J}=S_{J}^{-1}=\bigoplus_{j=1}^{r} \varepsilon_{j} F_{\ell_{j}} \oplus \bigoplus_{j=1}^{s} F_{2 m_{j}} .
$$

Here $\left\{\varepsilon_{1}, \ldots, \varepsilon_{r}\right\}$ with $\varepsilon_{j}= \pm 1$ is the sign characteristic associated with the real eigenvalues $\lambda_{j}, j=1: r$ of $P(\lambda)$. We easily check that $S_{J}=S_{J}^{*}$ and $J S_{J}=\left(J S_{J}\right)^{*}$. 
Appendix A.2. Real symmetric structure

Suppose $P(\lambda)$ is real symmetric with

- $r$ real elementary divisors $\left(\lambda-\lambda_{j}\right)^{\ell_{j}}, j=1: r$, and

- $s$ pairs of nonreal conjugate elementary divisors $\left(\lambda-\mu_{j}\right)^{m_{j}},\left(\lambda-\bar{\mu}_{j}\right)^{m_{j}}, j=1: s$

with $\ell_{j}, m_{j}$ such that $\sum_{j=1}^{r} \ell_{j}+2 \sum_{j=1}^{s} m_{j}=m n$. On using [9, Thm. 9.2] we find that

$$
J=\bigoplus_{j=1}^{r} J_{\ell_{j}}\left(\lambda_{j}\right) \oplus \bigoplus_{j=1}^{s} K_{2 m_{j}}\left(\mu_{j}, \bar{\mu}_{j}\right), \quad S_{J}=S_{J}^{-1}=\bigoplus_{j=1}^{r} \varepsilon_{j} F_{\ell_{j}} \oplus \bigoplus_{j=1}^{s} F_{2 m_{j}},
$$

where the scalars $\varepsilon_{j}= \pm 1$ form the sign characteristic associated with the real eigenvalues of $P(\lambda)$. Note that $S_{J}=S_{J}^{T}$ and $J S_{J}=\left(J S_{J}\right)^{T}$.

\section{Appendix A.3. Complex symmetric structure}

Suppose $P(\lambda)$ is complex symmetric with $q$ elementary divisors $\left(\lambda-\lambda_{j}\right)^{m_{j}}, \lambda_{j} \in \mathbb{C}$, $j=1: q$, with $m_{j}$ such that $\sum_{j=1}^{q} m_{j}=m n$. Then [20, Prop. 4.3] leads to

$$
J=\bigoplus_{j=1}^{q} J_{m_{j}}\left(\lambda_{j}\right), \quad S_{J}=S_{J}^{-1}=\bigoplus_{j=1}^{q} F_{m_{j}}
$$

which satisfy $S_{J}=S_{J}^{T}$ and $J S_{J}=\left(J S_{J}\right)^{T}$.

Appendix A.4. *-even structure

Suppose $P(\lambda)$ is *-even with

- $r$ purely imaginary (including 0 ) elementary divisors $\left(\lambda-i \beta_{j}\right)^{\ell_{j}}, j=1: r$, and

- $s$ pairs of nonzero and non-purely imaginary elementary divisors $\left(\lambda-i \mu_{j}\right)^{m_{j}},(\lambda-$ $\left.i \bar{\mu}_{j}\right)^{m_{j}}, j=1: s$

with $\ell_{j}, m_{j}$ such that $\sum_{j=1}^{r} \ell_{j}+2 \sum_{j=1}^{s} m_{j}=m n$. With the change of eigenvalue parameter $\lambda=-i \mu$, the $*$-even linearization of $P(\lambda), \lambda \mathcal{A}_{\mathcal{S}}+\mathcal{B}_{\mathcal{S}}=\mu\left(-i \mathcal{A}_{\mathcal{S}}\right)+\mathcal{B}_{\mathcal{S}}$ becomes a Hermitian pencil in $\mu$. Using Appendix A.1 we obtain that

$$
J=-i\left(\bigoplus_{j=1}^{r} J_{\ell_{j}}\left(-\beta_{j}\right) \oplus \bigoplus_{j=1}^{s}\left(J_{m_{j}}\left(-\bar{\mu}_{j}\right) \oplus J_{m_{j}}\left(-\mu_{j}\right)\right)\right), \quad S_{J}=-i\left(\bigoplus_{j=1}^{r} \varepsilon_{j} F_{\ell_{j}} \oplus \bigoplus_{j=1}^{s} F_{2 m_{j}}\right) .
$$

Here $\left\{\varepsilon_{1}, \ldots, \varepsilon_{r}\right\}$ with $\varepsilon_{j}= \pm 1$ is the sign characteristic associated with the zero and purely imaginary eigenvalues of $P(\lambda)$. Note that $S_{J}=-S_{J}^{*}$ and $J S_{J}=\left(J S_{J}\right)^{*}$.

\section{Appendix A.5. Real T-even structure}

Suppose $P(\lambda)$ is real $T$-even with (see [17])

- $t$ zero elementary divisors $\lambda^{n_{j}}$ with $n_{j}$ even, $j=1: t$,

- $r$ pairs of real elementary divisors $\left(\lambda+\alpha_{j}\right)^{p_{j}},\left(\lambda-\alpha_{j}\right)^{p_{j}}$ with $p_{j}$ odd if $\alpha_{j}=0$, $j=1: r$

- $s$ pairs of purely imaginary elementary divisors $\left(\lambda+i \beta_{j}\right)^{k_{j}},\left(\lambda-i \beta_{j}\right)^{k_{j}}$ with $\beta_{j}>0$, $j=1: s$, 
- $q$ quadruples of nonzero and non-purely imaginary elementary divisors $\left(\lambda+\mu_{j}\right)^{m_{j}},(\lambda-$ $\left.\mu_{j}\right)^{m_{j}},\left(\lambda+\bar{\mu}_{j}\right)^{m_{j}},\left(\lambda-\bar{\mu}_{j}\right)^{m_{j}}, j=1: q$,

with $n_{j}, p_{j}, k_{j}, m_{j}$ such that $\sum_{j=1}^{t} n_{j}+2 \sum_{j=1}^{r} p_{j}+2 \sum_{j=1}^{s} k_{j}+4 \sum_{j=1}^{q} m_{j}=m n$. Using [10, Thm. 16.1], we find that

$$
\begin{gathered}
J=\bigoplus_{j=1}^{t} J_{n_{j}}(0) \oplus \bigoplus_{j=1}^{r}\left(J_{p_{j}}\left(\alpha_{j}\right) \oplus-J_{p_{j}}\left(\alpha_{j}\right)^{T}\right) \\
\oplus \bigoplus_{j=1}^{s} K_{2 k_{j}}\left(i \beta_{j},-i \beta_{j}\right) \oplus \bigoplus_{j=1}^{q}\left(K_{2 m_{j}}\left(\mu_{j}, \bar{\mu}_{j}\right) \oplus-K_{2 m_{j}}\left(\mu_{j}, \bar{\mu}_{j}\right)^{T}\right), \\
S_{J}=\bigoplus_{j=1}^{t} \varepsilon_{j} E_{n_{j}} \oplus \bigoplus_{j=1}^{r}\left[\begin{array}{cc}
0 & -I_{p_{j}} \\
I_{p_{j}} & 0
\end{array}\right] \oplus \bigoplus_{j=1}^{s} \varepsilon_{j}\left(E_{k_{j}} \otimes E_{2}^{k_{j}}\right) \oplus \bigoplus_{j=1}^{q}\left[\begin{array}{cc}
0 & -I_{2 m_{j}} \\
I_{2 m_{j}} & 0
\end{array}\right],
\end{gathered}
$$

where the scalars $\varepsilon_{j}= \pm 1$ form the sign characteristic associated with the purely imaginary eigenvalues and zero eigenvalues of even partial multiplicities (see [19]). We easily check that $S_{J}=-S_{J}^{T}$ and $J S_{J}=\left(J S_{J}\right)^{T}$.

Appendix A.6. Complex T-even structure

Let $\lambda_{j} \in \mathbb{C} \backslash\{0\}$ and suppose $P(\lambda)$ is complex $T$-even with (see [17])

- $t$ zero elementary divisors $\lambda^{m_{j}}$ with $m_{j}$ even, $j=1: r$,

- $q$ pairs of elementary divisors $\left(\lambda-\lambda_{j}\right)^{k_{j}},\left(\lambda+\lambda_{j}\right)^{k_{j}}$ with $k_{j}$ odd if $\lambda_{j}=0, j=1: q$, with $m_{j}, k_{j}$ such that $\sum_{j=1}^{r} m_{j}+2 \sum_{j=1}^{q} k_{j}=m n$. Then, by [20, Prop. 4.7 (b)], we obtain that

$J=\bigoplus_{j=1}^{t} J_{m_{j}}(0) \oplus \bigoplus_{j=1}^{q}\left(J_{k_{j}}\left(\lambda_{j}\right) \oplus J_{k_{j}}\left(-\lambda_{j}\right)\right), \quad S_{J}=\bigoplus_{j=1}^{t}\left[\begin{array}{cc}0 & -F_{\frac{1}{2}} m_{j} \\ F_{\frac{1}{2} m_{j}} & 0\end{array}\right] \oplus \bigoplus_{j=1}^{q}\left[\begin{array}{cc}0 & -F_{k_{j}} \\ F_{k_{j}} & 0\end{array}\right]$.

Note that $S_{J}=-S_{J}^{T}$ and $J S_{J}=\left(J S_{J}\right)^{T}$.

Appendix A.7. *-odd structure

Suppose $P(\lambda)$ is *-odd with

- $r$ purely imaginary (including 0$)$ elementary divisors $\left(\lambda-i \beta_{j}\right)^{\ell_{j}}, j=1: r$ and

- $s$ pairs of nonzero and non-purely imaginary elementary divisors $\left(\lambda-i \mu_{j}\right)^{m_{j}},(\lambda-$ $\left.i \bar{\mu}_{j}\right)^{m_{j}}, j=1: s$,

with $\ell_{j}, m_{j}$ such that $\sum_{j=1}^{r} \ell_{j}+2 \sum_{j=1}^{s} m_{j}=m n$. Note that for the $*$-odd linearization $\lambda \mathcal{A}_{\mathcal{S}}+\mathcal{B}_{\mathcal{S}}$ of $P(\lambda)$ in $(4)$, the pencil $i\left(\lambda \mathcal{A}_{\mathcal{S}}+\mathcal{B}_{\mathcal{S}}\right)$ is $*$-even and the structure for $S_{J}$ and $J$ follows from Appendix A.4. We find that

$J=-i\left(\bigoplus_{j=1}^{r} J_{\ell_{j}}\left(-\beta_{j}\right) \oplus \bigoplus_{j=1}^{s}\left(J_{m_{j}}\left(-\bar{\mu}_{j}\right) \oplus J_{m_{j}}\left(-\mu_{j}\right)\right)\right), \quad S_{J}=S_{J}^{-1}=\bigoplus_{j=1}^{r} \varepsilon_{j} F_{\ell_{j}} \oplus \bigoplus_{j=1}^{s} F_{2 m_{j}}$,

which satisfy $S_{J}=S_{J}^{*}$ and $J S_{J}=-\left(J S_{J}\right)^{*}$. Here $\left\{\varepsilon_{1}, \ldots, \varepsilon_{r}\right\}$ with $\varepsilon_{j}= \pm 1$ is the sign characteristic associated with the zero and purely imaginary eigenvalues of $P(\lambda)$. 
Appendix A.8. Real T-odd structure

Suppose $P(\lambda)$ is real $T$-odd with (see [17])

- $t$ zero elementary divisors $\lambda^{\ell_{j}}$ with $\ell_{j}$ odd, $j=1: t$,

- $r$ pairs of real elementary divisors $\left(\lambda+\alpha_{j}\right)^{p_{j}},\left(\lambda-\alpha_{j}\right)^{p_{j}}$ with $p_{j}$ even if $\alpha_{j}=0$, $j=1: r$,

- $s$ pairs of purely imaginary elementary divisors $\left(\lambda+i \beta_{j}\right)^{k_{j}},\left(\lambda-i \beta_{j}\right)^{k_{j}}$ with $\beta_{j}>0$, $j=1: s$, and

- $q$ quadruples elementary divisors $\left(\lambda+\mu_{j}\right)^{m_{j}},\left(\lambda-\mu_{j}\right)^{m_{j}},\left(\lambda+\bar{\mu}_{j}\right)^{m_{j}},\left(\lambda-\bar{\mu}_{j}\right)^{m_{j}}$, $j=1: q$,

with $\ell_{j}, p_{j}, k_{j}, m_{j}$ such that $\sum_{j=1}^{t} \ell_{j}+2 \sum_{j=1}^{r} p_{j}+2 \sum_{j=1}^{s} k_{j}+4 \sum_{j=1}^{q} m_{j}=m n$. On using [10, Thm. 17.1] we find that

$$
\begin{gathered}
J=\bigoplus_{j=1}^{t} J_{\ell_{j}}(0) \oplus \bigoplus_{j=1}^{r}\left(J_{p_{j}}\left(\alpha_{j}\right) \oplus-J_{p_{j}}\left(\alpha_{j}\right)^{T}\right) \\
\oplus \bigoplus_{j=1}^{s} K_{2 k_{j}}\left(i \beta_{j},-i \beta_{j}\right) \oplus \bigoplus_{j=1}^{q}\left(K_{2 m_{j}}\left(\mu_{j}, \bar{\mu}_{j}\right) \oplus-K_{2 m_{j}}\left(\mu_{j}, \bar{\mu}_{j}\right)^{T}\right), \\
S_{J}=S_{J}^{-1}=\bigoplus_{j=1}^{t} \varepsilon_{j} E_{\ell_{j}} \oplus \bigoplus_{j=1}^{r}\left[\begin{array}{cc}
0 & I_{p_{j}} \\
I_{p_{j}} & 0
\end{array}\right] \oplus \bigoplus_{j=1}^{s} \varepsilon_{j}\left(E_{k_{j}} \otimes E_{2}^{k_{j}-1}\right) \oplus \bigoplus_{j=1}^{q}\left[\begin{array}{cc}
0 & I_{2 m_{j}} \\
I_{2 m_{j}} & 0
\end{array}\right],
\end{gathered}
$$

where the scalars $\varepsilon_{j}= \pm 1$ form the sign characteristic associated with the purely imaginary eigenvalues and the zero eigenvalues with odd partial multiplicities. We easily check that $S_{J}=S_{J}^{T}$ and $J S_{J}=-\left(J S_{J}\right)^{T}$.

\section{Appendix A.9. Complex T-odd structure}

Let $\lambda_{j} \in \mathbb{C} \backslash\{0\}$ and suppose $P(\lambda)$ is complex $T$-odd with (see [17])

- $s$ zero elementary divisors $\lambda^{\ell_{j}}$ with $\ell_{j}$ odd, $j=1: s$, and

- $q$ pairs of elementary divisors $\left(\lambda+\lambda_{j}\right)^{k_{j}},\left(\lambda-\lambda_{j}\right)^{k_{j}}$ with $k_{j}$ even if $\lambda_{j}=0, j=1: q$, with $\ell_{j}, k_{j}$ such that $\sum_{j=1}^{s} \ell_{j}+2 \sum_{j=1}^{q} k_{j}=m n$. It follows from [20, Prop. 4.7 (b)] that

$$
J=\bigoplus_{j=1}^{s} J_{\ell_{j}}(0) \oplus \bigoplus_{j=1}^{q}\left(-J_{k_{j}}\left(\lambda_{j}\right) \oplus J_{k_{j}}\left(\lambda_{j}\right)\right), \quad S_{J}=S_{J}^{-1}=\bigoplus_{j=1}^{s} E_{\ell_{j}} \oplus \bigoplus_{j=1}^{q} F_{2 k_{j}} .
$$

Clearly, $S_{J}=S_{J}^{T}$ and $J S_{J}=-\left(J S_{J}\right)^{T}$.

Notice the difference between the zero elementary divisors associated with $T$-even and $T$-odd pencils (see [17, Cor. 4.3]). 
Appendix A.10. *-(anti)palindromic structure

Suppose $P(\lambda)$ is complex $*$-palindromic with $-1 \notin \Lambda(P)$ and (see [18])

- $q$ pairs of elementary divisors $\left(\lambda-\lambda_{j}\right)^{k_{j}},\left(\lambda-1 / \bar{\lambda}_{j}\right)^{k_{j}}$ with $\lambda_{j} \in \mathbb{C},\left|\lambda_{j}\right| \neq 1, j=1: q$,

- $t$ elementary divisors $\left(\lambda-\lambda_{j}\right)^{2 \ell_{j}+1}$ with $\lambda_{j} \in \mathbb{C}$ such that $\left|\lambda_{j}\right|=1, j=1: t$, and

- $s$ elementary divisors $\left(\lambda-\lambda_{j}\right)^{2 m_{j}}$ with $\lambda_{j} \in \mathbb{C},\left|\lambda_{j}\right|=1, j=1: s$,

with $k_{j}, \ell_{j}, m_{j}$ such that $2 \sum_{j=1}^{q} k_{j}+\sum_{j=1}^{t}\left(2 \ell_{j}+1\right)+2 \sum_{j=1}^{s} m_{j}=m n$. Then using either [21, Thm. 5] or [22, Sec. 2.2.2] we find that

$$
J=-S_{J} S_{J}^{-*}
$$

with

$$
\begin{aligned}
S_{J} & =\bigoplus_{j=1}^{q}\left[\begin{array}{cc}
0_{k_{j}} & F_{k_{j}} J_{k_{j}}\left(-\lambda_{j}\right) \\
F_{k_{j}} & 0_{k_{j}}
\end{array}\right] \oplus \bigoplus_{j=1}^{t} \varepsilon_{j}\left[\begin{array}{ccc}
0 & 0 & F_{\ell_{j}} J_{\ell_{j}}\left(-\lambda_{j}\right) \\
0 & \left(-\lambda_{j}\right)^{1 / 2} & e_{1}^{T} \\
F_{\ell_{j}} & 0 & 0
\end{array}\right] \\
& \oplus \bigoplus_{j=1}^{s} \varepsilon_{j}\left[\begin{array}{cc}
0_{m_{j}} & F_{m_{j}} J_{m_{j}}\left(-\lambda_{j}\right) \\
F_{m_{j}} & e_{1} e_{1}^{T}
\end{array}\right],
\end{aligned}
$$

has the above elementary divisors. Here $e_{1}$ is the first column of the identity matrix. The scalars $\varepsilon_{j}= \pm 1$ form the sign characteristic associated with the eigenvalues of unit modulus of $P(\lambda)$ (see $[8])$.

For the *-antipalindromic structure, $J=S_{J} S_{J}^{-*}$ with $S_{J}$ as above but with $-\lambda_{j}$ replaced by $\lambda_{j}$.

\section{Appendix A.11. Real T-(anti)palindromic structure}

Suppose $P(\lambda)$ is real $T$-palindromic with $-1 \notin \Lambda(P), \lambda_{j} \in \mathbb{C} \backslash\{0\}$, and (see [18])

- $r$ pairs of real elementary divisors $\left(\lambda-\lambda_{j}\right)^{k_{j}},\left(\lambda-1 / \lambda_{j}\right)^{k_{j}}$ with $\lambda_{j} \in \mathbb{R},\left|\lambda_{j}\right| \neq 1$, $j=1: r$,

- $q$ quadruples of nonreal elementary divisors $\left(\lambda-\lambda_{j}\right)^{n_{j}},\left(\lambda-\bar{\lambda}_{j}\right)^{n_{j}},\left(\lambda-1 / \lambda_{j}\right)^{n_{j}}$, $\left(\lambda-1 / \bar{\lambda}_{j}\right)^{n_{j}}$ with $\left|\lambda_{j}\right| \neq 1, j=1: q$,

- $s$ elementary divisors $(\lambda-1)^{2 m_{j}}, j=1: s$,

- $t$ pairs of elementary divisors $(\lambda-1)^{2 \ell_{j}+1},(\lambda-1)^{2 \ell_{j}+1}, j=1: t$,

- $u$ pairs of elementary divisors $\left(\lambda-\lambda_{j}\right)^{\ell_{j}^{\prime}},\left(\lambda-\bar{\lambda}_{j}\right)^{\ell_{j}^{\prime}}$ with $\left|\lambda_{j}\right|=1, \lambda_{j} \neq 1, \ell_{j}^{\prime}$ odd, $j=1: u$

- $p$ pairs of elementary divisors $\left(\lambda-\lambda_{j}\right)^{m_{j}^{\prime}},\left(\lambda-\bar{\lambda}_{j}\right)^{m_{j}^{\prime}}$ with $\left|\lambda_{j}\right|=1, \lambda_{j} \neq 1, m_{j}^{\prime}$ even, $j=1: p$.

We have that $2 \sum_{j=1}^{r} k_{j}+4 \sum_{j=1}^{q} n_{j}+2 \sum_{j=1}^{s} m_{j}+2 \sum_{j=1}^{t}\left(2 \ell_{j}+1\right)+2 \sum_{j=1}^{u} \ell_{j}^{\prime}+$ $2 \sum_{j=1}^{p} m_{j}^{\prime}=m n$.

Using [22, Thm. 2.8] we find that $J=-S_{J} S_{J}^{-*}$ has the above list of elementary divisors, where

$$
S_{J}=\bigoplus_{j=1}^{r}\left[\begin{array}{cc}
0_{k_{j}} & F_{k_{j}} J_{k_{j}}\left(-\lambda_{j}\right) \\
F_{k_{j}} & 0_{k_{j}}
\end{array}\right] \oplus \bigoplus_{j=1}^{q}\left[\begin{array}{cc}
0_{2 n_{j}} & K_{2 n_{j}}\left(-\Lambda_{j}\right) \\
F_{n_{j}} \otimes I_{2} & 0_{2 n_{j}}
\end{array}\right] \oplus \bigoplus_{j=1}^{s}\left[\begin{array}{cc}
0 & F_{m_{j}} J_{m_{j}}(-1) \\
F_{m_{j}} & 0
\end{array}\right]
$$




$$
\begin{aligned}
\oplus \bigoplus_{j=1}^{t} \varepsilon_{j}\left[\begin{array}{ccc}
0_{\ell_{j}} & 0 & F_{\ell_{j}} J_{\ell_{j}}(-1) \\
0 & 1 & e_{1}^{T} \\
F_{\ell_{j}} & 0 & 0_{\ell_{j}}
\end{array}\right] \oplus \bigoplus_{j=1}^{t} \varepsilon_{j}\left[\begin{array}{ccc}
0_{\ell_{j}} & 0 & F_{\ell_{j}} J_{\ell_{j}}(-1) \\
0 & 1 & e_{1}^{T} \\
F_{\ell_{j}} & 0 & 0_{\ell_{j}}
\end{array}\right] \\
\oplus \bigoplus_{j=1}^{u} \varepsilon_{j}\left[\begin{array}{ccc}
0_{\ell_{j}^{\prime}-1} & 0 & K_{\ell_{j}^{\prime}-1}\left(-\Lambda_{j}\right) \\
0 & \left(-\Lambda_{j}\right)^{\frac{1}{2}} & e_{1}^{T} \otimes I_{2} \\
F_{\frac{1}{2}\left(\ell_{j}^{\prime}-1\right)} \otimes I_{2} & 0 & 0_{\ell_{j}^{\prime}-1}
\end{array}\right] \oplus \bigoplus_{j=1}^{p} \varepsilon_{j}\left[\begin{array}{cc}
0_{m_{j}^{\prime}} & K_{m_{j}^{\prime}}\left(-\Lambda_{j}\right) \\
F_{\frac{1}{2} m_{j}^{\prime}} \otimes I_{2} & e_{1} e_{1}^{T} \otimes I_{2}
\end{array}\right] .
\end{aligned}
$$

Here $\left(-\Lambda_{j}\right)^{\frac{1}{2}}$ is the principal square root of $-\Lambda_{j}$. The scalars $\varepsilon_{j}$ are signs \pm 1 and form the sign characteristic associated with the eigenvalues of unit modulus of $P(\lambda)$ except the eigenvalues 1 with even partial multiplicities (see [8]).

For the $T$-antipalindromic $P(\lambda), J=S_{J} S_{J}^{-T}$ where $S_{J}$ is as above but with $-\lambda_{j},-1$, $-\Lambda_{j}, 1$ replaced by $\lambda_{j}, 1, \Lambda_{j},-1$, respectively.

\section{Appendix A.12. Complex T-(anti)palindromic structure}

Suppose $P(\lambda)$ is complex $T$-palindromic with $-1 \notin \Lambda(P)$ and (see [18])

- $t$ elementary divisors $(\lambda-1)^{m_{j}}$ with $m_{j}$ even, $j=1: t$,

- $q$ pairs of elementary divisors $\left(\lambda-\lambda_{j}\right)^{k_{j}},\left(\lambda-1 / \lambda_{j}\right)^{k_{j}}$ with $k_{j}$ odd when $\lambda_{j}=1$, $j=1: q$,

with $m_{j}, k_{j}$ such that $\sum_{j=1}^{t} m_{j}+2 \sum_{j=1}^{q} k_{j}=m n$. On using either [21, Thm. 1] or [22, Thm. 2.6], we find that with

$$
S_{J}=\bigoplus_{j=1}^{t}\left[\begin{array}{cc}
0_{m_{j} / 2} & F_{m_{j} / 2} J_{m_{j} / 2}(-1) \\
F_{m_{j} / 2} & e_{1} e_{1}^{T}
\end{array}\right] \oplus \bigoplus_{j=1}^{q}\left[\begin{array}{cc}
0_{k_{j}} & F_{k_{j}} J_{k_{j}}\left(-\lambda_{j}\right) \\
F_{k_{j}} & 0_{k_{j}}
\end{array}\right]
$$

the matrix $J=-S_{J} S_{J}^{-T}$ has the above elementary divisors.

Now if $P(\lambda)$ is complex $T$-antipalindromic with $-1 \notin \Lambda(P)$ and (see [18])

- $t$ elementary divisors $(\lambda-1)^{\ell_{j}}$ with $\ell_{j}$ odd, $j=1: t$,

- $q$ pairs of elementary divisors $\left(\lambda-\lambda_{j}\right)^{k_{j}},\left(\lambda-1 / \lambda_{j}\right)^{k_{j}}$ with $k_{j}$ even if $\lambda_{j}=1$, $j=1: q$,

with $\ell_{j}, k_{j}$ such that $\sum_{j=1}^{t} \ell_{j}+2 \sum_{j=1}^{q} k_{j}=m n$. On using [22, Thm. 2.6], we find that the matrix $J=S_{J} S_{J}^{-T}$ with

$$
S_{J}=\bigoplus_{j=1}^{t}\left[\begin{array}{ccc}
0_{\ell_{j}} & 0 & F_{\ell_{j}} J_{\ell_{j}}(1) \\
0 & 1 & e_{1}^{T} \\
F_{\ell_{j}} & 0 & 0_{\ell_{j}}
\end{array}\right] \oplus \bigoplus_{j=1}^{q}\left[\begin{array}{cc}
0_{k_{j}} & F_{k_{j}} J_{k_{j}}\left(\lambda_{j}\right) \\
F_{k_{j}} & 0_{k_{j}}
\end{array}\right]
$$

has the above elementary divisors.

Note that $J$ in Appendix A.10-Appendix A.12 is "almost" in Jordan canonical form. 


\section{References}

[1] Maha Al-Ammari and Françoise Tisseur. Structured inverse quadratic eigenvalue problems. Technical report, Manchester Institute for Mathematical Sciences, The University of Manchester, UK, 2011. In preparation.

[2] Moody T. Chu and Shu-Fang Xu. Spectral decomposition of real symmetric quadratic $\lambda$-matrices and its applications. Math. Comp., 78:293-313, 2009.

[3] S. D. Garvey, P. Lancaster, A. A. Popov, U. Prells, and I. Zaballa. Filters connecting isospectral quadratic systems. To appear in Linear Algebra Appl., 2011.

[4] Israel Gohberg, Peter Lancaster, and Leiba Rodman. Spectral analysis of selfadjoint matrix polynomials. Ann. of Math. (2), 112(1):33-71, 1980.

[5] Israel Gohberg, Peter Lancaster, and Leiba Rodman. Indefinite Linear Algebra and Applications. Birkhäuser, Basel, Switzerland, 2005.

[6] Israel Gohberg, Peter Lancaster, and Leiba Rodman. Matrix Polynomials. Society for Industrial and Applied Mathematics, Philadelphia, PA, USA, 2009. Unabridged republication of book first published by Academic Press in 1982.

[7] Nicholas J. Higham, D. Steven Mackey, Niloufer Mackey, and Françoise Tisseur. Symmetric linearizations for matrix polynomials. SIAM J. Matrix Anal. Appl., 29(1):143-159, 2006.

[8] Peter Lancaster, Uwe Prells, and Leiba Rodman. Canonical structures for palindromic matrix polynomials. Oper. Matrices, 1(4):469-489, 2007.

[9] Peter Lancaster and Leiba Rodman. Canonical forms for Hermitian matrix pairs under strict equivalence and congruence. SIAM Rev., 47(3):407-443, 2005.

[10] Peter Lancaster and Leiba Rodman. Canonical forms for symmetric/skew-symmetric real matrix pairs under strict equivalence and congruence. Linear Algebra Appl., 406:1-76, 2005.

[11] Peter Lancaster and Miron Tismenetsky. The Theory of Matrices. Academic Press, London, second edition, 1985.

[12] Peter Lancaster and Ion Zaballa. A review of canonical forms for selfadjoint matrix polynomials. To appear in the Gohberg Memorial Volume. Springer, 2011.

[13] D. Steven Mackey. Structured Linearizations for Matrix Polynomials. PhD thesis, University of Manchester, Manchester, England, 2006.

[14] D. Steven Mackey. Quadratic realizability of matrix polynomials. Part I: The unstructured case. Manuscript, 2011.

[15] D. Steven Mackey, Niloufer Mackey, Christian Mehl, and Volker Mehrmann. Structured polynomial eigenvalue problems: Good vibrations from good linearizations. SIAM J. Matrix Anal. Appl., 28(4):1029-1051, 2006.

[16] D. Steven Mackey, Niloufer Mackey, Christian Mehl, and Volker Mehrmann. Vector spaces of linearizations for matrix polynomials. SIAM J. Matrix Anal. Appl., 28(4):971-1004, 2006.

[17] D. Steven Mackey, Niloufer Mackey, Christian Mehl, and Volker Mehrmann. Jordan structures of alternating matrix polynomials. Linear Algebra Appl., 432:867-891, 2010.

[18] D. Steven Mackey, Niloufer Mackey, Christian Mehl, and Volker Mehrmann. Smith forms of palindromic matrix polynomials. MIMS EPrint 2010.36, Manchester Institute for Mathematical Sciences, The University of Manchester, UK, May 2010.

[19] Volker Mehrmann and Hongguo Xu. Perturbation of purely imaginary eigenvalues of Hamiltonian matrices under structured perturbations. Electron. J. Linear Algebra, 17:234-257, 2008.

[20] Leiba Rodman. Comparison of congruences and strict equivalences for real, complex, and quaternionic matrix pencils with symmetries. Electron. J. Linear Algebra, 16:248-283, 2007.

[21] Christian Schröder. A canonical form for palindromic pencils and palindromic factorizations. Technical Report No. 316, DFG Research Center, MATHEON, Technische Universität Berlin, Berlin, Germany, 2006.

[22] Christian Schröder. Palindromic and Even Eigenvalue Problems - Analysis and Numerical Methods. PhD thesis, Technischen Universitat Berlin, Germany, 2008. 